\title{
Ultraweitwinkel-Fundusfotografie und -angiografie in der Differenzialdiagnose und zur Therapieplanung bei peripheren vaskulären Netzhauterkrankungen
}

\section{Ultra-Wide Field Retinal Imaging and Angiography in the Differential Diagnosis and Therapeutic Decisions in Vascular Diseases of the Peripheral Retina}

\author{
Autoren \\ Bert Müller ${ }^{1}$ \\ Institute \\ 1 Klinik und Poliklinik für Augenheilkunde, Charité - \\ Universitätsmedizin Berlin, Berlin \\ 2 Berlin Institute of Health (BIH), Berlin
}

Antonia M. Joussen ${ }^{1,2}$, Claudia Brockmann ${ }^{1,2}$, Josefine Urban ${ }^{1}$, Ira Seibel ${ }^{1,2}$, Sibylle Winterhalter ${ }^{1}$, Oliver Zeitz ${ }^{1,2}$,

\section{Schlüsselwörter}

familiär exsudative Vitreoretinopathie, Hämangioblastom, Morbus Coats, vasoproliferative retinale Tumoren, Weitwinkelangiografie, Weitwinkelbildgebung

Key words

familiar exudative vitreoretinopathy, hemangioblastoma, coats disease, vasoproliferative retinal tumor, ultra-wide field angiography, ultra-wide field imaging

eingereicht $\quad 15.6 .2018$

Bibliografie

DOI https://doi.org/10.1055/a-0667-0493

Klin Monatsbl Augenheilkd 2018; 235: 980-993 @ Georg

Thieme Verlag KG Stuttgart · New York | ISSN 0023-2165

Korrespondenzadresse

Univ.-Prof. Dr. med. Antonia M. Joussen

Charité - Universitätsmedizin Berlin, Klinik und Poliklinik

für Augenheilkunde

Augustenburger Platz 1, 13353 Berlin

Hindenburgdamm 30, 12203 Berlin

Tel.: + 49(0) 3084452331 , Fax: + 49(0) 3084454450

antonia.joussen@charite.de

\section{ZUSAMMENFASSUNG}

Bei vaskulären Erkrankungen der Netzhautperipherie ist eine Bildgebung, die einen umfassenden Einblick in die periphere Netzhaut ermöglicht und diese reproduzierbar abbilden kann, von hoher klinischer Relevanz. Die Weitwinkelangiografie ist in der Differenzialdiagnose peripherer vaskulärer Netzhaut- erkrankungen insbesondere zur Therapieentscheidung und Verlaufsbeurteilung wertvoll. Da Erkrankungen wie Morbus Coats und die familiär exsudative Vitreoretinopathie meist innerhalb der 1. oder Anfang der 2. Lebensdekade diagnostiziert werden, ist bei den meisten Patienten mit unzureichender Compliance bei Fundusaufnahmen zu rechnen. Gerade hier ermöglicht die Ultraweitwinkelbildgebung eine deutlich kürzere Aufnahmezeit im Vergleich zu Kompositaufnahmen. Dennoch können auch die aktuellsten Systeme nicht die gesamte Netzhaut vollständig abbilden. Der Bildausschnitt muss daher gezielt durch Blickrichtungen zum betroffenen Areal gelenkt werden und dem Fotografen die klinische Fragestellung stets zu Beginn der Aufnahmen bewusst sein. Daher steht nach wie vor eine gründliche Funduskopie in Mydriasis durch den erfahrenen Augenarzt an erster Stelle.

\section{ABSTRACT}

Investigation of vascular diseases of the peripheral retina requires imaging procedures that allow a comprehensive view of the periphery, as well as reproducible pictures. In particular, ultra-wide field fluorescence angiography facilitates diagnosis, therapeutic decisions and follow-up examinations. While vasculopathies such as Coats disease and familiar exudative vitreoretinopathy are diagnosed within the first and second decade of life, patients' compliance during fundus imaging is typically reduced within this age range. Compared to the repeated imaging procedures for composite formation, ultra-wide field imaging has significantly reduced recording time. Nevertheless, current imaging systems are not able to map the entire retina in scaled proportions. Therefore, the imaging frame must be guided by patients' gaze onto the affected retinal area. Moreover, the medical photographer must be aware of the clinical setting and the region of interest. Hence, previous detailed funduscopy by trained ophthalmologists will remain indispensable. 


\section{Einleitung}

Die präzise Diagnostik sehr peripher gelegener Netzhautveränderungen ist eine der wesentlichen Herausforderungen der klinischen Augenheilkunde. Die vorliegende Übersicht beschäftigt sich mit sehr peripher gelegenen vaskulär-proliferativen sowie exsudativen Erkrankungen der peripheren Netzhaut und diskutiert, wie die neue Ultraweitwinkelbildgebung das klinische Management dieser Erkrankungen beeinflusst.

Bei den kleinsten Patienten mit Frühgeborenenretinopathie (ROP) ist die Weitwinkelbildgebung bereits seit vielen Jahren ein zentrales diagnostisches Instrument, unter anderem auch zur Dokumentation. Bei ROP steht für die Untersuchung am Frühgeborenen und bei Kindern in den ersten Lebensjahren mit der RetCam (Clarity Medical Systems Inc.) ein Verfahren zur Verfügung, das mit direktem Hornhautkontakt im Säuglings- und Kinderauge die Netzhautperipherie darstellen kann und auch eine Angiografie ermöglicht [1].

Beim Erwachsenen haben Ultraweitwinkelsysteme die Darstellung des Ausmaßes der (peripheren) retinalen Ischämie und peripher proliferativer Veränderungen stark vereinfacht. Dies kommt z.B. Patienten mit exsudativen Veränderungen wie bei Morbus Coats und familiär exsudativer Vitreoretinopathie (FEVR) zugute. Anwendungen finden sich auch bei Patienten mit Morbus Eales und anderen „entzündlichen“ Ischämien [2]. Durch die Non-Kontakt-Ultraweitwinkelbildgebung sind Verlaufsuntersuchungen insbesondere im Kindesalter deutlich patientenfreundlicher geworden und ersparen den Patienten Untersuchungen in Allgemeinnarkose. Bemerkenswerte Befunde konnten an klinisch asymptomatischen Partneraugen von FEVR- und Morbus-CoatsPatienten erhoben werden: Nicht selten finden sich in der Ultraweitwinkelbildgebung subklinische periphere Netzhautveränderungen (Brockmann, zur Publikation eingereicht).

Bevor auf die genannten Erkrankungen im Einzelnen eingegangen wird, sollen im Folgenden allgemeine und technische Überlegungen dargestellt werden.

\section{Allgemeine und technische Überlegungen}

Gerade bei Erkrankungen der peripheren Netzhaut hat die Ultraweitwinkelbildgebung eine hohe klinische Bedeutung, weil die relevanten Befunde einfach sichtbar gemacht und dokumentiert werden können. Weitwinkeldarstellungen fanden im Alltag auch vor der Einführung ausgeklügelter Bildgebungssysteme unter Nutzung eines Mainster-Glases statt - allerdings als Kontaktmethode. Die aktuell zur Verfügung stehenden Systeme von Optos und Zeiss verwenden unterschiedliche Methoden der Darstellung der Peripherie mittels Scan und Spiegel oder als Kombination aus Fundusfoto und Scan. Details sind in den verschiedenen Beiträgen dieses Schwerpunktthemas zu finden.

Während die Funduskopie durch den erfahrenen Augenarzt sicher der Goldstandard bleibt, erlauben digitale UltraweitwinkelBildgebungssysteme eine multimodale Bildgebung der äußersten Netzhautperipherie und damit eine bequeme Befunddokumentation. Insbesondere im Zeitalter elektronischer Patientendokumentationssysteme sind die ausführlichen peripheren Fundusskizzen zum Teil in den elektronischen Systemen nur noch schwer zu erstellen, hingegen die Einbindung von Bildmaterialien in die elektronische Krankenakte leichter möglich. Da moderne Geräte ohne großen Aufwand die periphere Netzhaut jenseits von $120^{\circ}$ darstellen können, hat sich die Bildgebung gegenüber den früher zum Teil händisch zusammenzusetzenden Aufnahmen (sogenannte Komposits) deutlich vereinfacht. Erst diese Vereinfachung ermöglicht longitudinale Aufnahmen bei akzeptabler Patientenund Mitarbeiterbelastung.

Insgesamt sind die aktuell verfügbaren Verfahren im Bereich der vaskulären Erkrankungen der peripheren Netzhaut in der Hauptsache zur Dokumentation augenärztlich erhobener Befunde, jedoch eher nicht als Screeningmethode geeignet. Dies ist der Tatsache geschuldet, dass selbst die Ultraweitwinkelbildgebung keine absolut vollständige Abbildung der kompletten Netzhautperipherie in einer Aufnahme ermöglicht.

Es können neben dem Colorbild auch sehr periphere Angiografien oder Autofluoreszenzbilder gewonnen werden. Diese Aufnahmen sind nicht nur in der primären Diagnostik relevant, sondern auch in der Verlaufsbeurteilung nach Therapie. Mittels Angiografie können periphere ischämische Netzhautareale sichtbar und in gewissen Grenzen auch quantifiziert werden [3]. Zudem kommen periphere Leckagen oder Neovaskularisationen zur Darstellung. Dies ist insbesondere zur Einschätzung der Krankheitsaktivität und von Krankheitsverläufen mit und ohne Therapie relevant. Im Gegensatz dazu spielt die Autofluoreszenz bei den peripheren retinal-vaskulären Veränderungen in der Regel keine Rolle, es sei denn, es kommt zu einer Mitbeteiligung des RPE (retinales Pigmentepithel) wie beispielsweise im Falle einer Narbenbildung. Die multimodale Darstellung (IR [Infrarot]/AF [Autofluoreszenz]/ FAG [Fluoreszenzangiografie]) ist bei der Ultraweitwinkelbildgebung in einem Aufnahmegang möglich. In Zukunft werden die Systeme noch um eine optische Kohärenztomografie (OCT) der Netzhautperipherie ergänzt werden. Ebenfalls wird die Zukunft funktionelle Bildgebungsverfahren ermöglichen, sodass beispielsweise eine periphere Netzhauthypoxie dargestellt werden kann.

Die Schwächen der aktuellen Ultraweitwinkelsysteme sind auch bekannt: Die Ultraweitwinkelbildgebung kann zwar über $200^{\circ}$ der Peripherie abbilden, aber es entstehen Verzerrungen nach oben und unten. Wenn die Lider nicht herausgehalten werden können, sind häufig Zilien auf dem Bild sichtbar. Die Bildgebung z.B. mit dem Optos-Gerät benötigt einen sehr dichten Kontakt zum Auge, was bei tiefliegenden Augen die Bildgebung erschweren kann.

Die Falschfarbendarstellung einiger Systeme (Optomap) kann die Interpretation der Bilder zum Teil erschweren. Eine funduskopische Untersuchung kann hier häufig die korrekte Einschätzung des Bildes erleichtern (siehe auch Abb. 2 im Artikel Nürnberg et al. [4] in diesem Heft: Hier ist eine Glaskörperverdichtung gezeigt, die sich wie eine Pigmentierung darstellt.).

Im folgenden Abschnitt soll die Weitwinkeldiagnostik in der Differenzialdiagnose und zum Therapiemonitoring verschiedener Krankheitsbilder eingeordnet werden. 




\section{Retinopathia praematurorum (ROP)}

Eine Weitwinkelbildgebung im Bereich der Frühgeborenentherapie und -diagnostik wird nun schon längere Zeit mit der RetCam genutzt. Die RetCam ist eine handgehaltene Kamera, die in Kontakt mit der Kornea eine Darstellung der peripheren Netzhaut im Säuglings- und Kinderauge ermöglicht. Zur Dokumentation und Inspektion der peripheren Netzhaut muss gegebenenfalls zusätzlich eine Indentation vorgenommen werden. Die Nutzung der RetCam unter telemedizinischen Aspekten durch die behandelnde Neonatologie mag in Pilotprojekten gelungen sein, ist sicher aber nicht als Standard anzusehen. Die funduskopische Inspektion durch den erfahrenen Retinologen ist weiterhin ein integraler Bestandteil des Frühgeborenenscreenings unter Nutzung der RetCam als Dokumentationstool.

Ähnlich wie bei der ROP hat sich die Weitwinkeldiagnostik auch im frühen Kindesalter zu einem wichtigen Dokumentationstool in der Retinologie etabliert. Die Kombination der Nutzung der RetCam bei Narkoseuntersuchung und die frühe Gewöhnung der Kinder an die Optos-Weitwinkelfotografie ermöglicht eine objektive Befunddokumentation, die durch Zeichnungen ergänzt werden kann.

Mit der Einführung der Anti-VEGF-Therapie bei zentraler ROP ist es erstmalig möglich, im Gegensatz zur Laserphotokoagulation, die retinale Vaskularisation der Netzhautperipherie zu beeinflussen und zumindest passager ein Wachstum der Gefäßfront über die Leiste hinaus in die Peripherie zu begünstigen und zugleich eine Regression der Neovaskularisationen zu erzielen [5]. In $>$ Abb. 1 ist ein Patientenbeispiel zu sehen, bei dem 3 Monate nach intravitrealer Anti-VEGF-Therapie mit Bevacizumab ein merklicher Rückgang der prominenten Leiste zu verzeichnen war.

- Abb. 2 und $\mathbf{3}$ zeigen, wie bei dem gleichen Patienten nach 6 Jahren eine fast vollständige Vaskularisierung der ehemals avas- kulären Peripherie erreicht werden konnte. Behandlungsbedürftige Rezidive in weiter peripher gelegenen Regionen werden in der Literatur mit 10-15\% nach 3 Monaten angegeben. Sehr selten (Einzelfälle) werden auch späte Rezidive mit Netzhautablösung bis zu 3 Jahre nach der Erstmanifestation angegeben. Unterschwellige Rezidive ohne Progression bilden sich erfahrungsgemäß spontan zurück [6, 7]. Dennoch verbleiben häufig periphere Netzhautanteile scheinbar avaskulär, ein Befund, den man toleriert, aber aus bislang ungeklärter klinischer Relevanz und vor dem Hintergrund möglicher Spätrezidive mit Netzhautablösung in regelmäßigen Abständen kontrolliert [8, 9]. Im Gegensatz dazu konnte mit einer Lasertherapie der ischämischen avaskulären Peripherie bei zentralen ROP-Stadien nur eine sehr kleine Netzhautrestinsel erhalten werden, die Gefäße wuchsen nicht über die pathologische Leiste in die Peripherie hinaus. A Abb. 4 zeigt Weitwinkelaufnahmen eines Kindes nach Laserung einer ROP nach zentraler Zone-2-Erkrankung.

Frühgeburtlichkeit, ROP und Laserkoagulation begünstigen über die vaskuläre Akuterkrankung hinaus die Entstehung hochgradiger Myopien mit ausgeprägten peripheren vitreoretinalen Degenerationen, die zeitlebens ein Risiko für Netzhautablösung darstellen und ebenfalls regelmäßige Funduskontrollen erfordern [10-12]. In > Abb. 5 ist ein heute jugendlicher Patient mit ehemaliger ROP 3 und spontaner Regression zu sehen, der jetzt hoch myop ist und in der Peripherie ausgedehnte Netzhautdegenerationen (Schneckenspuren) aufweist.

Wie oben beschrieben können mit dem Ultraweitwinkelsystem von Optos Kinder schon im Kleinkindalter mit etwas Übung untersucht werden, limitierend kann insbesondere bei ehemaligen Frühgeborenen ein ausgeprägter Nystagmus sein oder ein ausgeprägtes Aufmerksamkeitsdefizit. Mit etwas Übung ist die Dokumentation der peripheren Netzhaut aber durchaus ab dem 4. Lebensjahr möglich. 


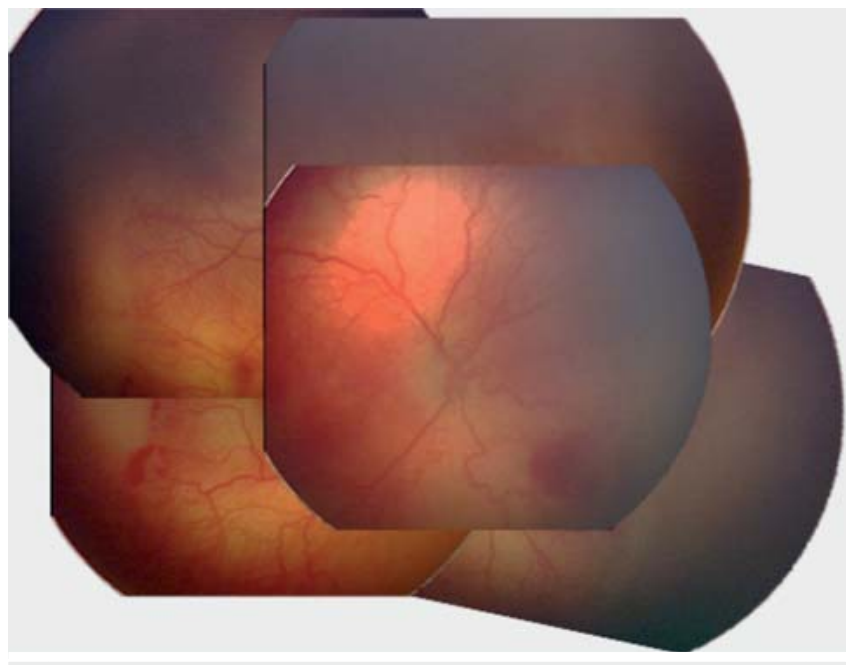

- Abb. 2 Frühgeborenenretinopathie. Rechtes Auge mit zentraler Erkrankung in Zone 2 (Komposit aus RetCam-Bildern).

\section{Familiär exsudative Vitreoretinopathie (FEVR)}

FEVR wurde vielfach inkorrekt als ROP ohne Frühgeburtlichkeit beschrieben [13]. Diese Beschreibung fußt auf der Tatsache, dass beide Erkrankungen mit einer ischämischen Netzhautperipherie und einer Verziehung der Gefäßstraßen nach temporal und damit mit einer Verkleinerung des Winkels к zwischen den Gefäßstraßen einhergehen [14]. Bei ausgeprägter peripherer Traktion kann eine falciforme Veränderung entstehen ( $\bullet$ Abb. 6). Kommt es zu einer zusätzlichen peripheren Exsudation ( $\bullet$ Abb. 7), so kann eine falciforme Ablatio entstehen, bei welcher die stabile exzentrische Fixation neben der Falte durch eine Zunahme der subretinalen Flüssigkeit und Verbreiterung der Falte dann verloren geht.

Im Gegensatz zur ROP beinhaltet die primäre Gefäßpathologie bei der FEVR neben der peripheren Ischämie die Exsudation (wie bei Morbus Coats). Dieser folgen sekundäre Veränderungen des Netzhautgewebes und des Glaskörpers. Exsudation markiert stets eine Krankheitsprogression und ist Indikator für einen Therapiebedarf. Alle Stadien mit Exsudation haben (weiteren) Therapiebedarf, ebenso wie extensive periphere avaskuläre Bereiche. Ziel der Therapie ist in Analogie zum Morbus Coats eine Zerstörung der pathologischen Gefäße und eine sekundäre Minderung der Leckage. Hierzu ist eine disseminierte Koagulation der ischämischen Peripherie bzw. bei peripheren Exsudationen eine Kryotherapie, die gegebenenfalls mehrere Male in Abständen von 6-8 Wochen wiederholt werden muss, notwendig (vgl. > Abb. 7). Die Weitwinkelbildgebung kann in der Indikationsstellung und in der Dokumentation des Behandlungserfolgs relevant sein, erfordert aber wie bei Morbus Coats eine (gegebenenfalls schwierig einzuhaltende) Blickrichtung während der Fotografie nach temporal und temporal unten, um die Krankheitsaktivität zu überwachen.

Bei progredienten falciformen Ablationes muss die Traktion durch eine indentierende Maßnahme reduziert werden, in der Regel wird hier temporal eine limbusparallele Plombe gelegt. Die Vitrektomie sollte, wenn irgendwie möglich, wegen der Veränderung der Glaskörperkonsistenz mit Insertionen in die retinale Glia vermieden werden.

Die bekannten Mutationen finden sich auf 5 Genorten. Es sind unter anderem autosomal-dominante, aber auch x-chromosomal-
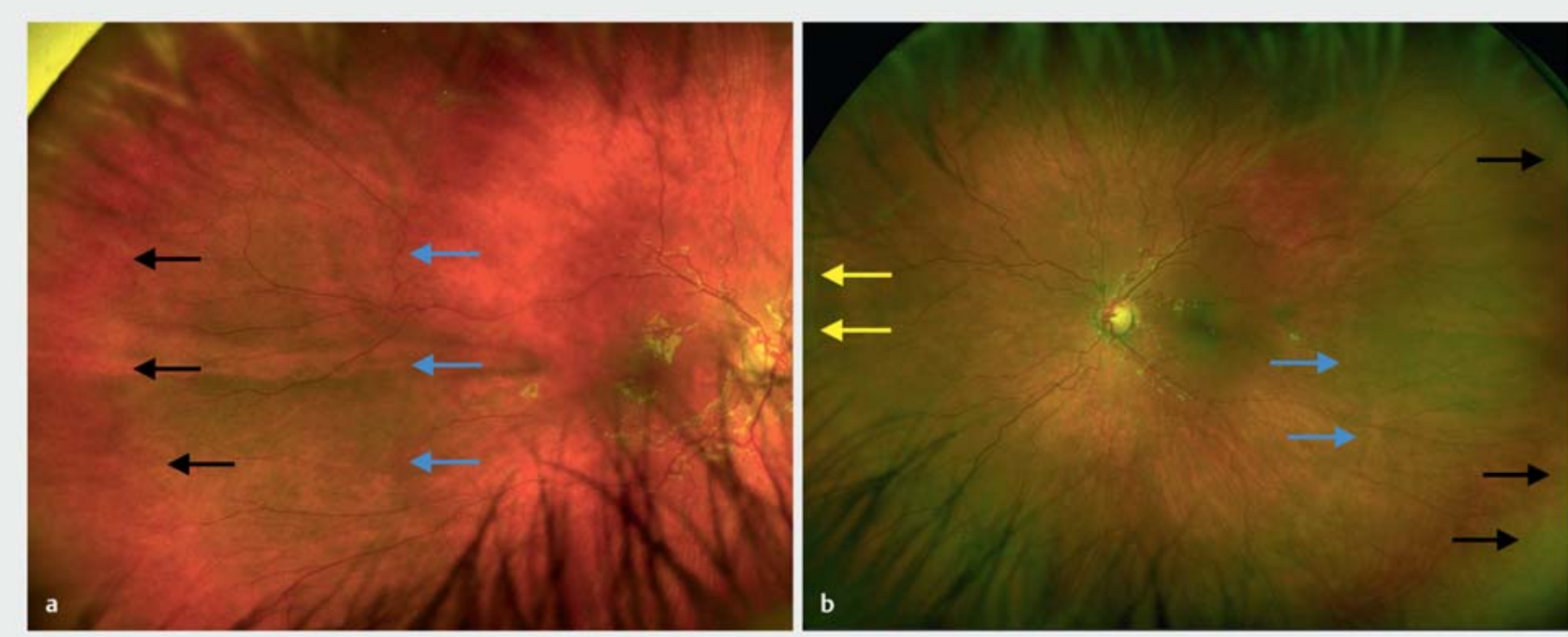

- Abb. 3 Frühgeborenenretinopathie. a Rechtes Auge 6 Jahre nach intravitrealer Injektion von Bevacizumab: Die initiale prominente Leiste ist als feine Linie innerhalb des Netzhautgewebes zu sehen (blaue Pfeile), die retinalen Gefäße sind bis weit in die Peripherie gewachsen (schwarze Pfeile). Sehr weit peripher davon ist noch eine schmale avaskuläre Zone zu sehen. b Linkes Auge, ebenfalls 6 Jahre nach intravitrealer Injektion von Bevacizumab: die retinalen Gefäße sind auch hier über die ehemalige Leiste (blaue Pfeile) bis weit in die Peripherie gewachsen (schwarze Pfeile). Nach nasal ist die Adhärenz des Glaskörpers sehr gut sichtbar (gelbe Pfeile). 


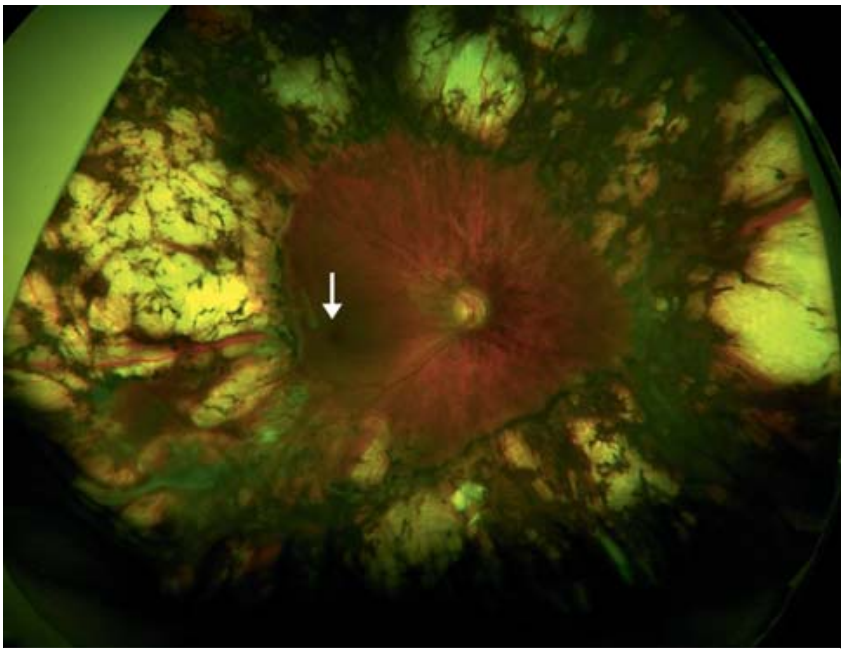

- Abb. 4 Frühgeborenenretinopathie. Rechtes und einziges Auge eines heute 9-jährigen Jungen mit Z.n. Laserkoagulation, ehemals zentraler Zone-2-Erkrankung. Die retinalen Gefäße konnten nicht über die Leiste in die Peripherie wachsen, das daraus resultierende Gesichtsfeld ist nur sehr klein. Der weiße Pfeil markiert die Fovea.

rezessive Erbgänge bekannt. Mittels Weitwinkelangiografie sollten auch Familienmitglieder, die potenziell betroffen sind, oder Genträger untersucht werden. Die Ausprägung der Erkrankung kann zwischen Familienmitgliedern sehr unterschiedlich sein. Die FEVR ist eine lebenslängliche Erkrankung, bei welcher die Weitwinkelbildgebung genauer als die früheren Fundusskizzen und aufwendigen Kompositaufnahmen den Krankheitsverlauf und den Therapieeffekt in einer vergleichbaren und standardisierten Form festhalten kann. Die dafür notwendigen Blickrichtungen fanden bereits Erwähnung.

\section{Morbus Coats und andere exsudative Vaskulopathien}

Die charakteristischen peripheren Mikroaneurysmen und Teleangiektasien in Verbindung mit gelblichen Exsudationen sind charakteristisch für Morbus Coats [13]. Während in fortgeschrittenen Stadien die Therapie lediglich einen Augenerhalt zum Ziel haben kann, ist eine Behandlung in frühen Stadien mit dem Ziel des Funktionserhalts besonders relevant [15].

Die Ultraweitwinkelangiografie erlaubt eine detaillierte Darstellung der peripheren Gefäßpathologie für die Therapieplanung. Um eine ausreichende Regression der pathologischen Gefäße zu erlauben, müssen alle pathologischen peripheren Gefäße koaguliert werden. Hierbei werden die Gefäße, anders als bei der Koagulation von peripher ischämischen Arealen wie beim Morbus Eales oder Susac-Syndrom, gezielt und direkt mit langen Pulsen koaguliert. Ziel ist der Gefäßverschluss und die Okklusion der peripher pathologischen Kapillaren. Gelingt die Koagulation der Gefäßpathologien, kommt es zu einer langsamen Regression der peripheren Exsudation. Hierzu ist ein Abtransport durch Makrophagen und Mikrogliazellen erforderlich, der Zeiträume von mehre- ren Monaten in Anspruch nehmen kann. Auch zentrale Exsudate, welche durch die peripheren Gefäßpathologien gespeist werden, sind so rückbildungsfähig. - Abb. 8 zeigt einen Morbus Coats mit peripher großflächigen Ischämien, die disseminiert koaguliert werden müssen, aber auch bis nach zentral gehende Gefäßpathologien, die einer direkten Koagulation bedürfen. Wie bei anderen exsudativen Gefäßerkrankungen kann auch beim Morbus Coats eine Anti-VEGF-Therapie die Exsudation supprimieren und so die Koagulation der Gefäßpathologien erleichtern. Die Anti-VEGFTherapie allein kann aber keine dauerhafte Regression der Exsudationen oder der zugrunde liegenden Gefäßpathologien bewirken. Nur die Koagulation der peripheren Gefäßveränderungen kann die Krankheitsprogression verhindern. Auch im Erwachsenenalter kann ein unzureichend behandelter Morbus Coats noch zu einer exsudativen Ablatio oder zur Entstehung von peripheren vasoproliferativen Tumoren (siehe unten) führen mit Folgen für den Visus und teils schlechter Prognose für den Augenerhalt.

Wie bei der FEVR ist bei Morbus Coats eine sorgfältige langfristige Beobachtung erforderlich, die auch das nicht oder milder betroffene Partnerauge miteinschließen muss. Während im Kleinkindesalter bei noch aktiven Erkrankungsstadien eine Narkoseuntersuchung unabdingbar ist, können ältere Kinder im Verlauf zum Teil schon mit einer Ultraweitwinkelbildgebung ohne Narkose untersucht werden. Dies stellt einen klaren und wichtigen Fortschritt dar. Besonders bedeutsam ist es allerdings, immer alle Bereiche der peripheren Netzhaut ausreichend untersucht zu haben, insbesondere zur Untersuchung der superioren und inferioren Netzhaut müssen hier entsprechende Blickrichtungen eingenommen werden. Die Ultraweitwinkelbildgebung ergänzt, aber ersetzt die klinische Funduskopie mit geeigneten Lupen nicht.

\section{Vasoproliferative retinale Tumoren}

Vasoproliferative retinale Tumoren (VPRT) sind meist kugelförmige, gelb-weißliche Veränderungen der peripheren Netzhaut, die amelanotisch und meist von geringer Prominenz sind [16]. Die Läsionen können aber über den Verlauf von Monaten eine Wachstumstendenz zeigen und werden dann durch die assoziierte BlutRetina-Schranken-Störung klinisch symptomatisch. Es können neben gelblichen Exsudaten Makulaödeme, Glaskörpertrübungen und im Verlauf reaktive epiretinale Membranen auftreten [17].

Die Bezeichnung „vasoproliferativer Tumor“ ist aus histologischer Sicht nicht präzise, da es sich nicht um primäre Gefäßtumoren, sondern um Tumoren handelt, die überwiegend aus Gliazellen (charakteristisch ist der immunhistochemische Nachweis von GFAP [Glial fibrillary acidic Protein]) und zusätzlich aus proliferierenden retinalen Pigmentepithelzellen aufgebaut sind [18-20], sodass VPRT von einigen Autoren auch als „reactive retinal Glioangiosis" klassifiziert werden [21]. VPRT sind eher wenig bis kaum vaskularisiert, erweiterte „Feeder Vessel“ wie bei kapillären Hämangiomen (Hämangioblastomen) kommen nicht vor.

Der Begriff Tumor wird häufig als irreführend empfunden, so handelt es sich um eine gutartige Läsion. Die Bezeichnung „vasoproliferativer Tumor“ wurde von Shields eingeführt, nachdem eine Vielzahl von Bezeichnungen für dieses Krankheitsbild („presumed acquired Hemangioma of the Retina“, „peripheral nodular 

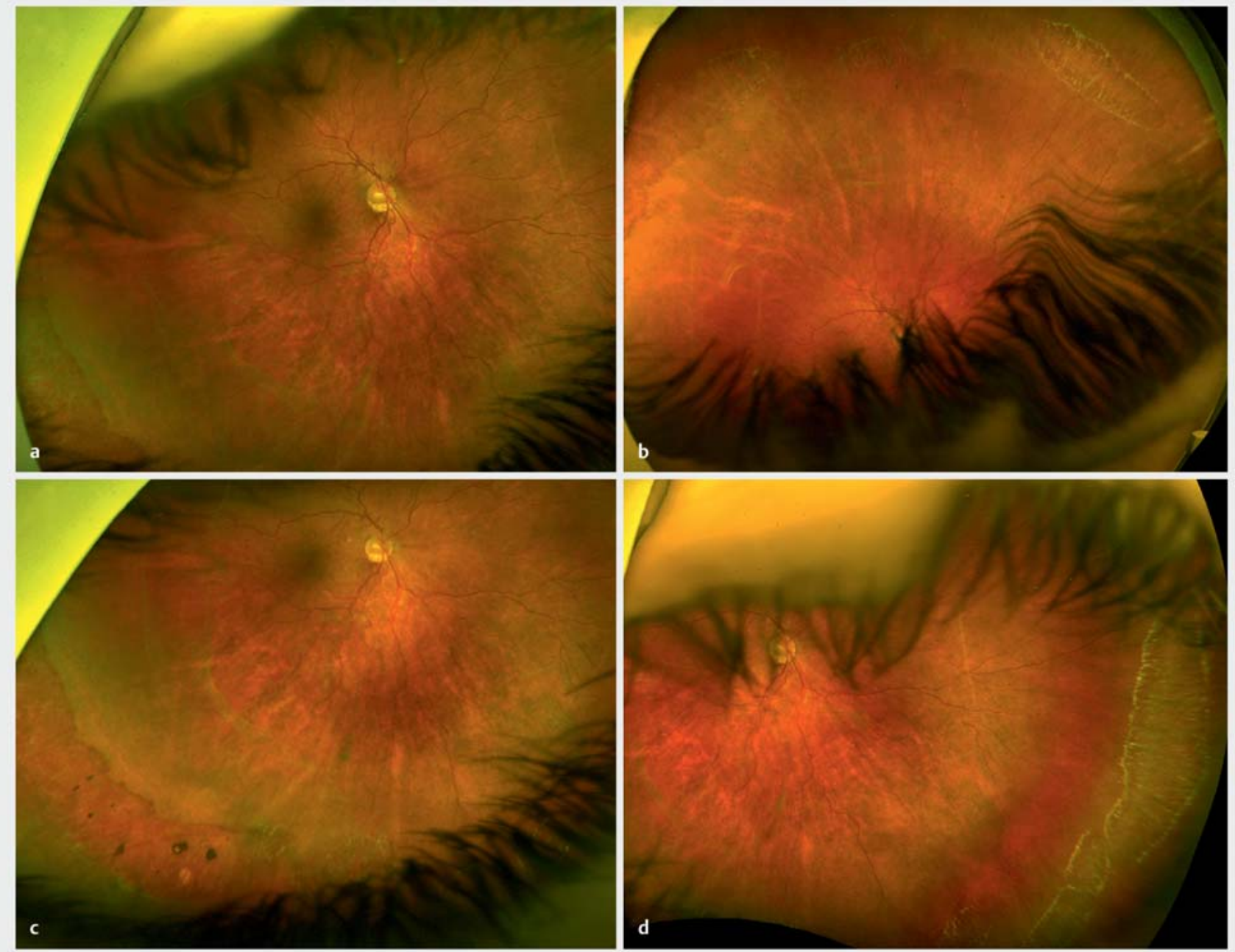

- Abb. 5 Frühgeborenenretinopathie. Rechtes Auge eines heute 15-jährigen Jungen (geboren in der 24. Schwangerschaftswoche) mit hoher Myopie von - $10 \mathrm{dpt}$ und Z. n. Threshold ROP (Retinopathy of Prematurity) mit spontaner Regression. Sehr anschaulich sind hier die peripheren Schneckenspurdegenerationen. a, c Rechtes Auge. b, d Linkes Auge.

Telangiectasis“ oder „Alters-Coats“) synonym in der Literatur gebraucht wurden [22, 23].

Recht häufig tritt ein vasoproliferativer Tumor als Sekundärveränderung auf, wie beispielsweise bei einem peripheren Morbus Coats ( $\triangleright$ Abb.9), aber auch bei Retinitis pigmentosa oder nach Kryotherapie mit Buckelchirurgie. Letzteres könnte ein Indiz dafür sein, dass die Entstehung des vasoproliferativen Tumors im Sinne einer Vernarbungsreaktion ähnlich einer proliferativen Vitreoretinopathie (PVR) zu werten sein könnte, wie andere Autoren schon gemutmaßt haben [24].

Unbehandelt führen VPRT zu einer zunehmenden exsudativen oder traktiven Netzhautablösung mit Visusverlust und Sekundärglaukom, sodass VPRT spätestens bei Auftreten einer Symptomatik behandelt werden sollten. Lokale Behandlungsmethoden stehen dabei im Vordergrund. Kleine Tumoren bis ca. 1,5 mm Prominenz können mit transkonjunktivaler Kryotherapie effektiv behandelt werden. Hierbei können mehrere Therapiesitzungen er- forderlich sein. Bei größeren Tumoren kann die Brachytherapie die Zerstörung des Tumors mit Erhalt der zentralen Netzhautfunktion erreichen, wobei $\beta$-Applikatoren $\left({ }^{106} \mathrm{Ru} /{ }^{106} \mathrm{Rh}\right)$ wegen der günstigeren Dosisverteilung am geeignetsten erscheinen [18, 25 , 26]. Die primäre Erfolgsrate (Inaktivierung der exsudativen Aktivität) nach Ruthenium-Applikator bei vasoproliferativem retinalem Tumor beträgt $70 \%$, bei erneuter exsudativer Aktivität konnte auch nach adjuvanter Therapie nur in $25 \%$ ein stabiler Befund erreicht werden [27].

- Abb. 9 zeigt die Narbe nach Applikator bei einem 35-jährigen Patienten. In der Weitwinkelbildgebung stellt sich dar, dass in den angrenzenden Bereichen der Netzhaut de novo auftretende Coats-artige Gefäße zu finden sind.

Die Therapie mit VEGF-Inhibitoren kann nicht als kausal angesehen werden, sondern führt lediglich zu einer symptomatischen Reduktion der Leckage der pathologischen Gefäße. Das Tumorvolumen nimmt ebenfalls ab, sodass auch größere Tumoren dann 

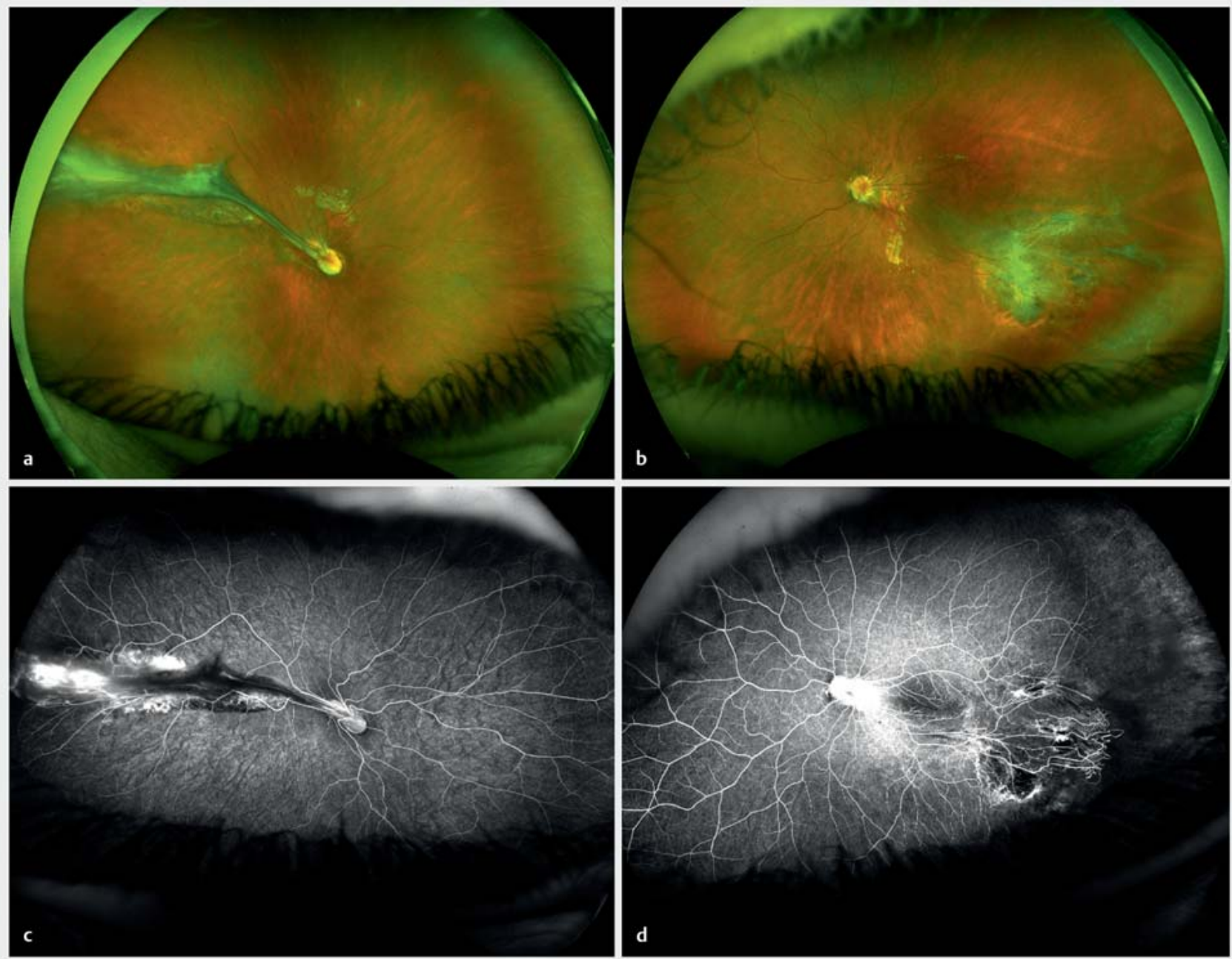

- Abb. 6 Familiäre exsudative Vitreoretinopathie. Beidseitiger Befund, am rechten Auge mit deutlichen falciformen Verziehungen nach temporal (a und c), am linken Auge sind die ausgeprägten peripheren avaskulären Bereiche sehr gut zu erkennen (b und d).

einer Kryotherapie zugänglich gemacht werden können. Die Injektion der VEGF-Inhibitoren muss gegebenenfalls repetitiv erfolgen. Die anschließende tumordestruktive Therapie sollte im Intervall stattfinden. Additiv sind eine Kryotherapie bei kleinem Tumorvolumen oder eine Bestrahlung mittels Brachytherapie notwendig ( $>$ Abb. 10).

Bornfeld und Mitarbeiter konnten zeigen, dass vasoproliferative Tumoren am häufigsten im temporal unteren Quadranten zu finden sind [25]. Daher ist für die Darstellung der Blick in die betreffende Richtung wichtig, ein Screening mit Weitwinkelbildgebung würde die Tumoren nicht darstellen. Die Weitwinkelbildgebung kann aber in der Dokumentation der differenzialdiagnostischen Überlegungen helfen: Hier müssen kapilläre Hämangiome unterschieden werden, wobei die hier bestehenden großvolumigen zu- und abführenden Gefäße (Feeder Vessel) in der Regel richtungsweisend sind. Eine molekulargenetische Zusatzuntersuchung ist hier in aller Regel nicht erforderlich. Die periphere exsudative hämorrhagische Chorioretinopathie (PEHCR) (siehe Arti- kel Seibel et al. [28] in diesem Heft) tritt überwiegend in höherem Lebensalter auf und ist neben ihrer Bilateralität meist mit anderen AMD-typischen Veränderungen vergesellschaftet.

Astrozytome wären eher am hinteren Augenpol lokalisiert, amelanotische Melanome der Uvea sind im Unterschied zum VPRT in der Aderhaut lokalisiert.

\section{Periphere vaskuläre Tumoren}

Die im Bereich der Netzhaut des Auges auftretenden Hämangioblastome werden häufig fälschlicherweise als retinale Angiome bezeichnet. Retinale Hämangioblastome sind häufig das erste Zeichen (in 50\% der Fälle multipel und bilateral). In der Regel sind sie symptomlos, können aber Netzhautablösung, Makulaödem, Glaukom und Visusverlust hervorrufen. Die Sonderform des juxtapapillären Hämangioms kann mit einer massiven Exsudation einhergehen. 

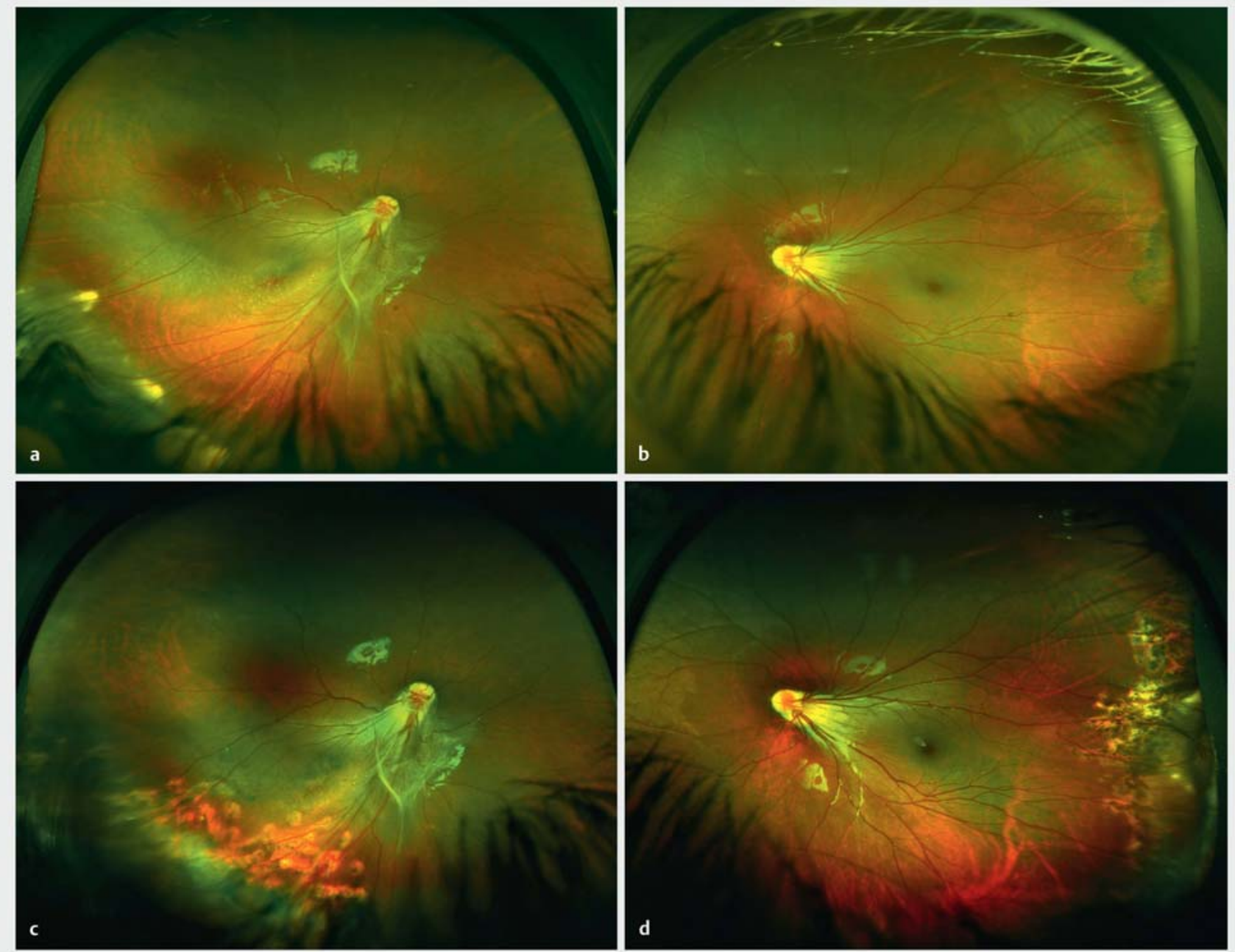

- Abb. 7 Familiäre exsudative Vitreoretinopathie. Beidseitiger Befund mit falciformen Verziehungen nach temporal und peripheren Exsudationen vor Therapie ( $\mathbf{a}$ und $\mathbf{b}$ ). An beiden Augen erfolgten bereits Kryo- und Laserkoagulationen (c und d), weitere Kontrollen inklusive Koagulationen sind erforderlich.

Hämangioblastome sind in etwa der Hälfte aller Fälle mit dem von-Hippel-Lindau-Syndrom (VHL) assoziiert, können aber auch sporadisch auftreten (50\%). Sporadische und hereditäre Angiome können klinisch nicht differenziert werden. VHL ist autosomaldominant vererbt; $80 \%$ ererbt von einem betroffenen Elternteil, $20 \%$ De-novo-Mutationen [29].

Hämangioblastome des zentralen Nervensystems werden als gutartige Tumoren nach der WHO-Klassifikation der Tumoren des zentralen Nervensystems als Grad 1 eingeteilt. Sie sind häufig im Bereich des Kleinhirns, des Hirnstamms oder des Rückenmarks lokalisiert, kommen selten aber auch im Großhirn vor [30]. Auch in weiteren Organen haben VHL-Patienten ein Risiko für die Entstehung von Tumoren. Betroffene Patienten ererben eine mutierte Kopie des VHL-Gens über die Keimbahn. Im späteren Leben erfolgt eine somatische Mutation (2. Treffer) des VHL-Gens in empfänglichen Geweben, die eine lokale Tumorgenese initiiert. Die Folge sind Nierenzellkarzinome, Nebennieren-, Nebenhoden- und Pankreastumoren [31].
Die Therapie kleiner peripherer retinaler Hämangioblastome kann in der Regel mit einer Laser- oder Kryokoagulation erfolgreich durchgeführt werden. Zentralere Tumoren, prominentere Läsionen oder Läsionen im Bereich einer bereits abgehobenen Netzhaut stellen therapeutische Herausforderungen dar ( $\bullet$ Abb. 11). Bestrahlungen mittels Brachytherapie oder Protonentherapie sind beschrieben, ebenso wie chirurgische Verfahren [32,33]. Die Hämangioblastome weisen häufig im Bereich der Feeder Vessels eine gliös-traktive Komponente auf, die chirurgisch entlastet werden kann. Die chirurgische Resektion bleibt Einzelfällen vorbehalten, in der Hälfte der Fälle ist die Entstehung neuer Läsionen an anderer Stelle nach Resektion beschrieben [32]. 

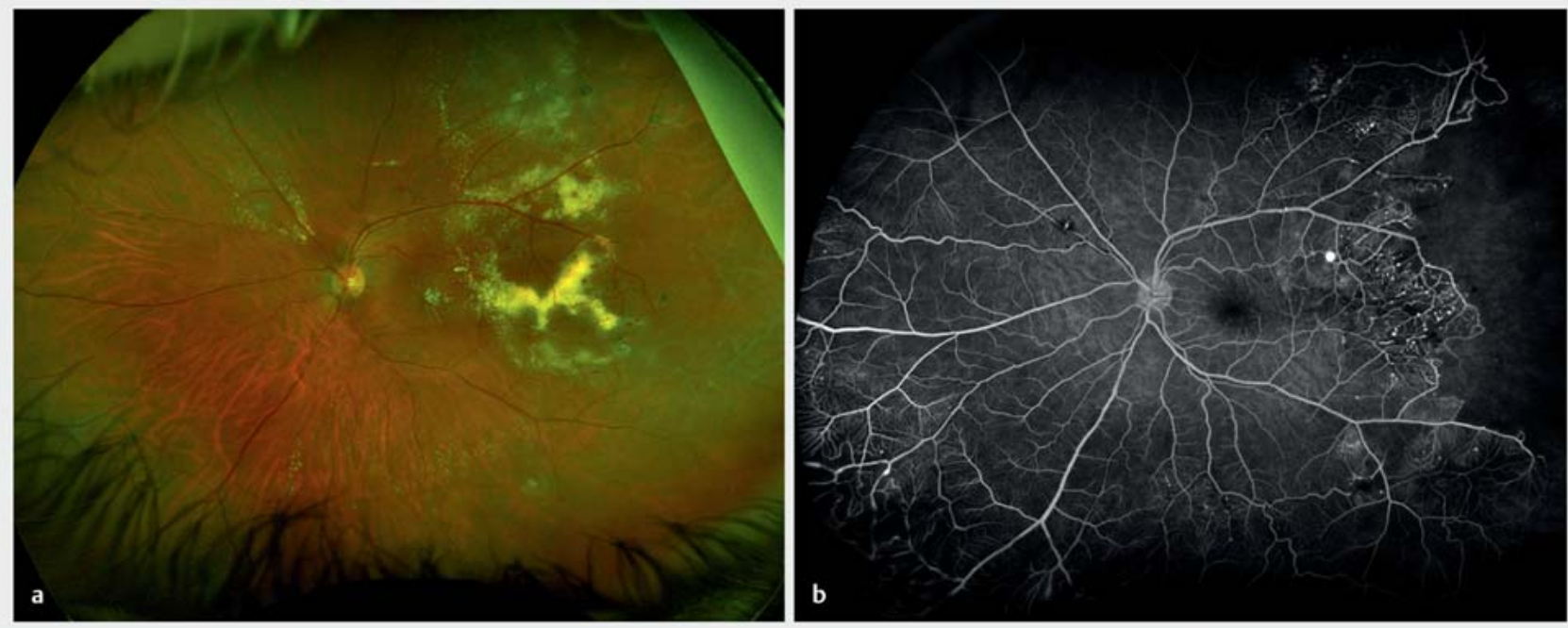

- Abb. 8 Morbus Coats. Linkes Auge im Stadium 2B mit massiven gelben Exsudaten mit korrespondierenden Mikroaneurysmata, bis nach zentral reichend (a) und ausgedehnten ischämischen Arealen nach temporal in der Fluoresceinangiografie (b).
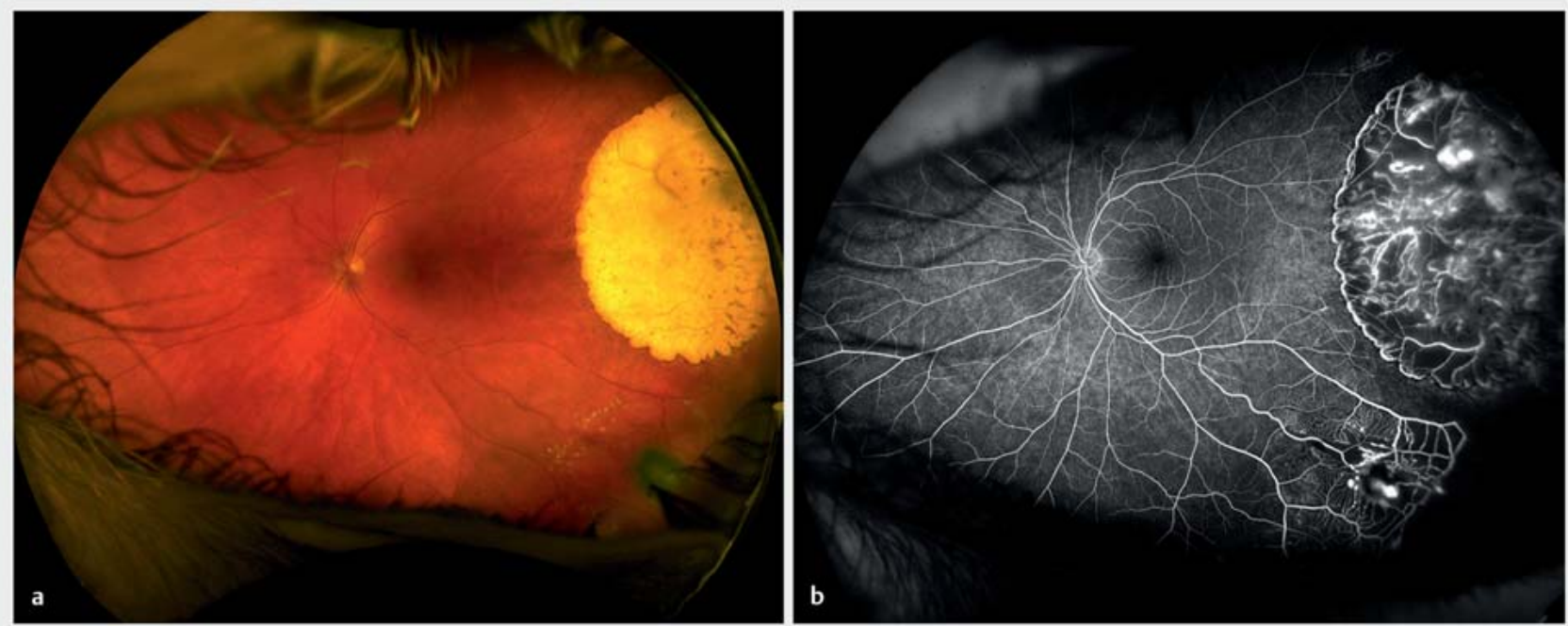

- Abb. 9 Morbus Coats mit sekundärem vasoproliferativem Tumor. Linkes Auge, das mittels Ruthenium-Applikator vor Jahren behandelt wurde, weist eine markante Strahlennarbe auf (a). Inferior davon zeigen sich weitere vaskuläre Auffälligkeiten, welche in der Fluoresceinangiografie besonders gut zur Darstellung kommen (b).

\section{Vaskuläre Anomalien und kavernöse Hämangiome}

Zu unterscheiden von den kapillären Hämangiomen sind die kavernösen Hämangiome und die arteriovenösen Malformationen. Sowohl bei kavernösen Hämangiomen wie bei den racemösen Angiomen sollte eine zerebrale Beteiligung ausgeschlossen werden.

Diagnostisch richtungsweisend ist die Ultraweitwinkelangiografie, die im Verlauf ein partielles Auswaschen des Fluoresceins aus den kavernösen Veränderungen zeigt, das charakteristisch für diese intraretinalen kapillären Aussackungen ist. Die typischen kappenartigen Füllungsdefekte sind nicht mit Leckagen assoziiert ( Abb. 12). Ein Therapiebedarf besteht bei kavernösen Hämangiomen in der Regel nicht.

Anders ist dies bei racemösen Angiomen, die durch retinale arteriovenöse Kommunikationen entstehen ( $\mathbf{A b b}$. 13). Es handelt sich um Phakomatosen, bei denen häufig die retinalen arteriovenösen Kommunikationen mit ipsilateralen zerebralen arteriovenösen Kommunikationen verbunden sind. Die Nomen- 

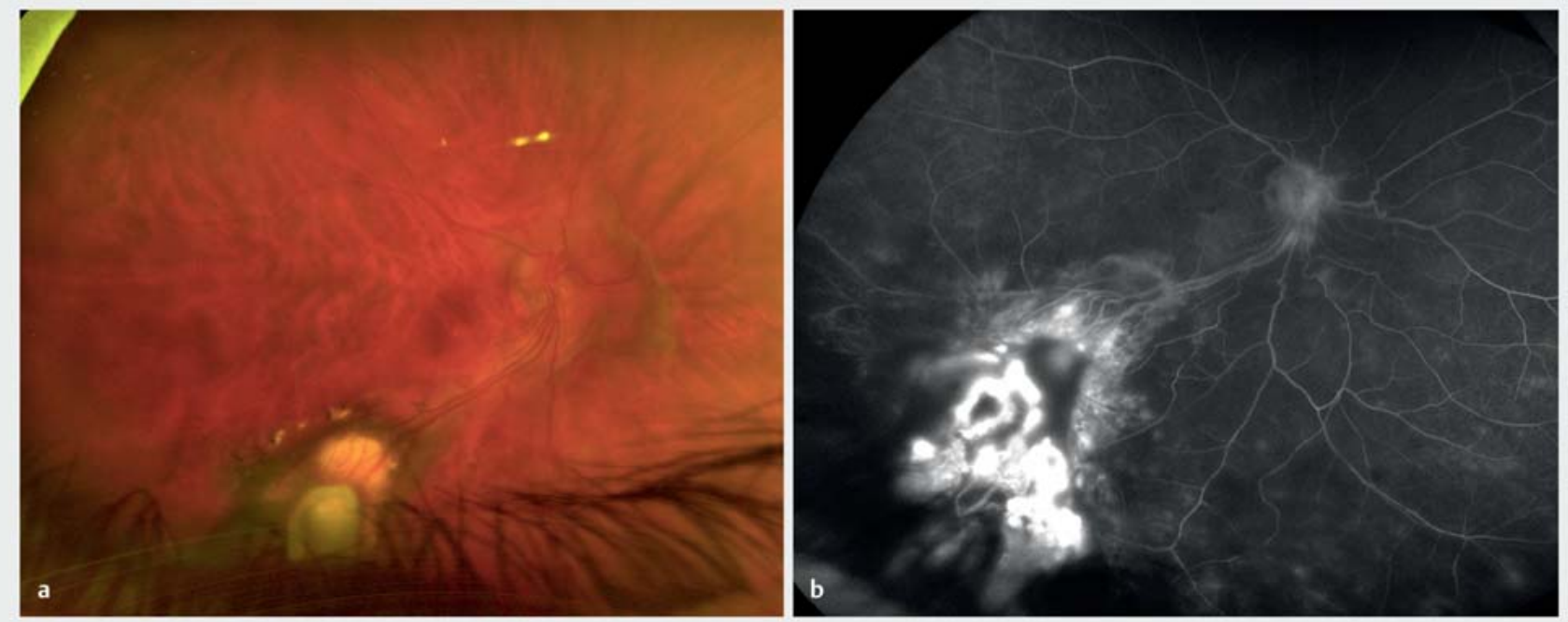

- Abb. 10 Primärer vasoproliferativer Tumor. Rechtes Auge mit einer prominenten Läsion nach temporal inferior. Die charakteristischen gliotischen Proliferationen und Exsudationen dieser sehr weit peripher gelegenen Läsionen sind in der Weitwinkeloptik inklusive Angiografie gut zugänglich (a und b). Bei diesem Auge erfolgte eine Brachytherapie mittels Ruthenium-Applikator.
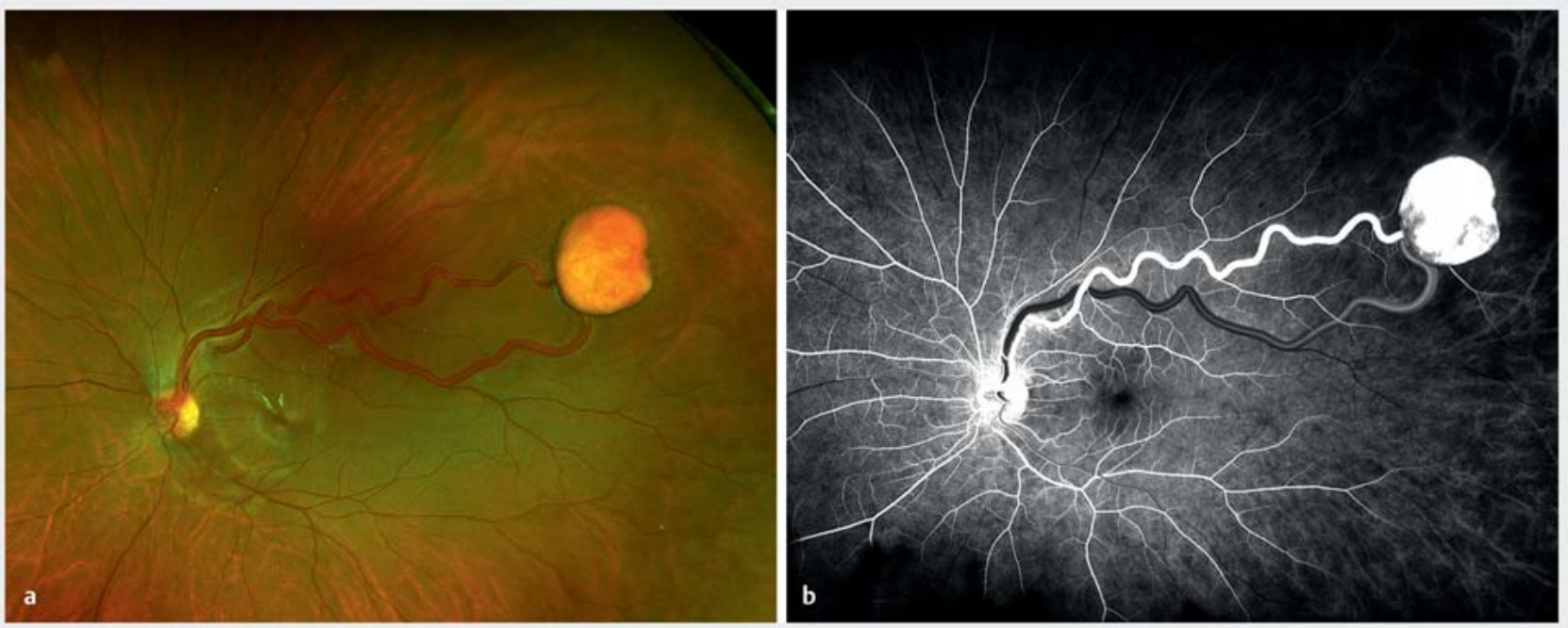

- Abb. 11 Retinales kapilläres Hämangioblastom. Linkes Auge mit großer, kugeliger Gefäßanomalie in der mittleren Peripherie nach temporal superior. Das zu- und abführende Gefäß stammt jeweils aus dem großen Gefäßbogen temporal superior (a). In der frühen Phase der Fluoreszenzangiografie kann man den direkten arteriellen Zustrom unmittelbar verfolgen (b).

klatur ist heterogen: Congenital unilateral retinocephalic vascular Malformation Syndrome (CRC Syndrome), kongenitales okulozerebrales Syndrom, Arteriovenous Communications of the Retina (AVCR). In der Augenheilkunde am weitesten verbreitet ist die Bezeichnung Wyburn-Mason-Syndrom.

Wichtig ist bei augenärztlicher Erstdiagnose die Abklärung der zerebralen Beteiligung. Ophthalmologisch werden diese Patienten durch Komplikationen der großkalibrigen High-Flow-Gefäße auffällig. Häufig sind Venenthrombosen beschrieben, die durch gemeinsame Gefäßscheiden zu erklären sind [34]. Während die arteriovenösen Kommunikationen therapeutisch nicht beeinflussbar sind und auch in Ausprägung und Lokalisation im Verlauf von Jahren variieren können, sollten die Komplikationen wie venöse Verschlüsse therapiert werden, um die Endstadien eines rubeotischen Glaukoms zu verhindern. Hierbei kann zur Reduktion einer ischämischen Reaktion sowohl eine Photokoagulation der peripheren ischämischen Netzhaut (im Gegensatz zu exsudativ vaskulären Veränderungen wie Morbus Coats oder FEVR nicht der pa- 

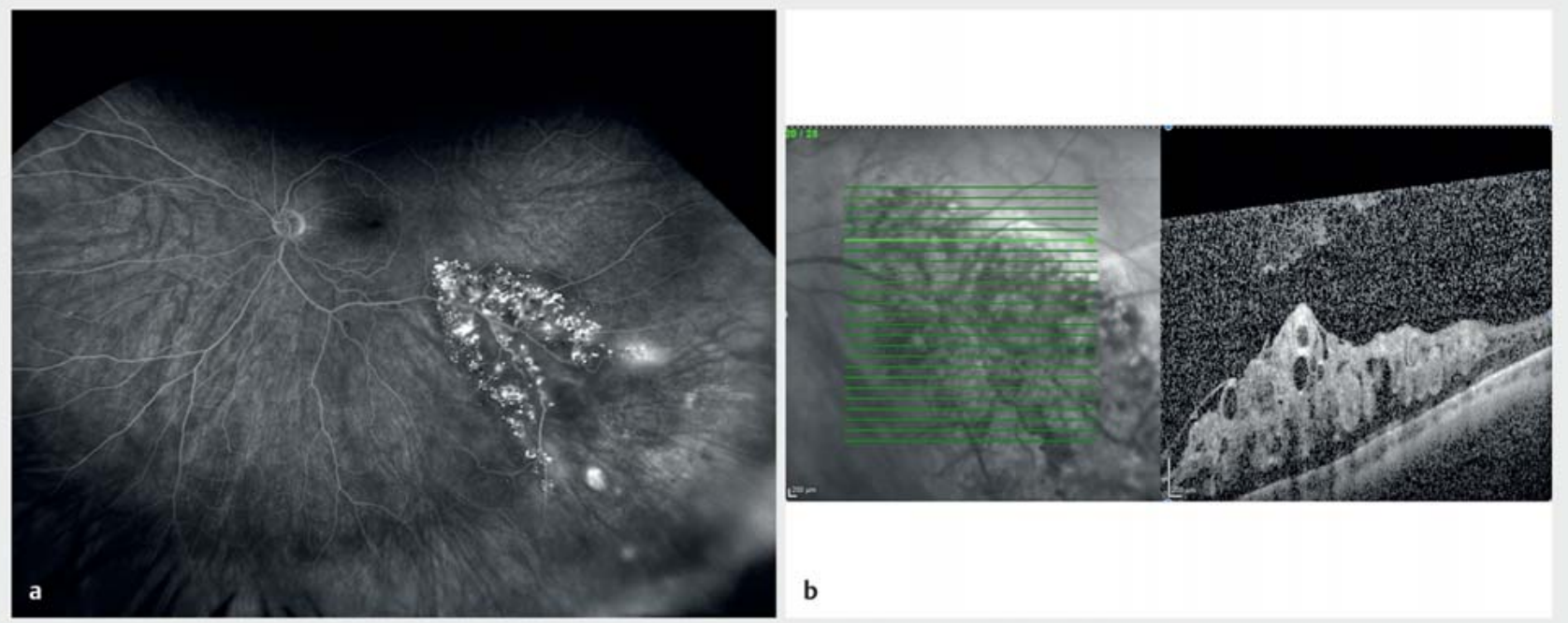

- Abb. 12 Retinales kavernöses Hämangiom. Linkes Auge mit kavernöser Läsion nach temporal inferior. In der Fluoreszenzangiografie sieht man die typischen kappenartigen Füllungsdefekte ohne Leckage (a), die optische Kohärenztomografie zeigt die kavernösen Aussackungen (b).

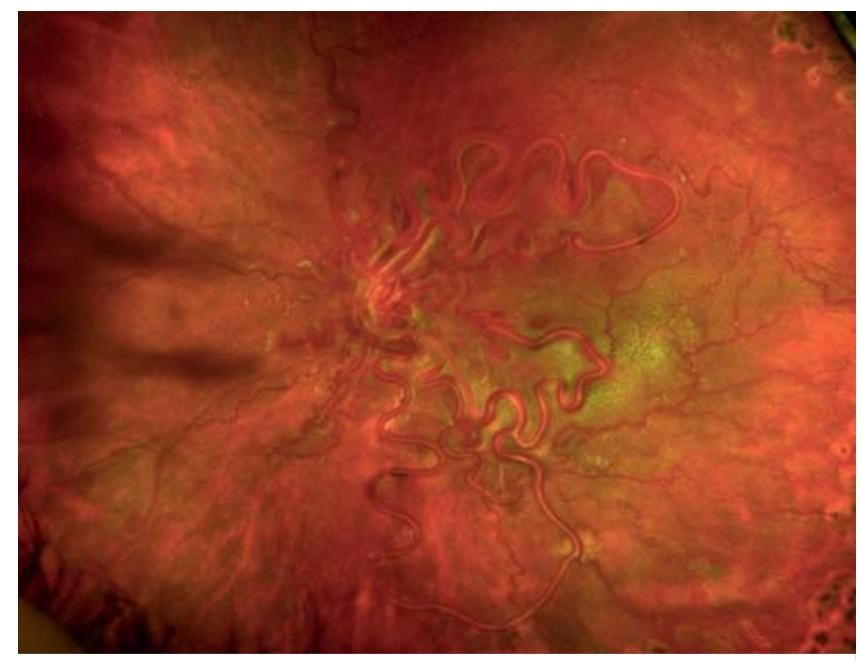

- Abb. 13 Racemöses Angiom bei Wyburn-Mason-Syndrom. Linkes Auge eines 6-jährigen Mädchens mit ausgeprägten retinalen arteriovenösen Kommunikationen im Zentrum und deutlich dilatierten, großkalibrigen Gefäßen. Es zeigt sich wenig Exsudation, peripher wurde bei auffälligen gittrigen Degenerationen koaguliert.

thologischen Gefäße) als auch eine Anti-VEGF-Therapie erfolgen $(\checkmark$ Abb. 14). Bei der Anti-VEGF-Therapie ist das oft jugendliche Alter der Patienten zu beachten, auch im Hinblick auf die Therapiedauer. Es kann dabei aber keine Änderung der arteriovenösen Kommunikationen durch die Anti-VEGF-Therapie erwartet werden.

\section{Morbus Eales und periphere entzündliche Ischämien wie das Susac-Syndrom}

Der Blick in die äußere Peripherie lohnt sich bei Erkrankungen, die mit einer okklusiv entzündlichen Veränderung der äußeren Netzhautperipherie einhergehen.

Henry Eales (1852-1913), ein englischer Augenarzt, beschrieb 1880 die „Angiopathia retinae juvenilis“: „Young men with epistaxis, constipation and headache, suffering vitreous bleeding“. Heute ist die Erkrankung als Morbus Eales bzw. Periphlebitis retinae bekannt. Insbesondere in den frühen Erkrankungsstadien stehen periphere Gefäßeinscheidungen und Ischämien im Vordergrund, sodass eine entzündliche infektiöse Genese angenommen wurde, eine schlüssige Korrelation, beispielsweise mit einer Tuberkulose, konnte nicht gezeigt werden [35]. Während also weiterhin die Ätiologie unbekannt ist, kommt es in den vergangenen Jahren doch wieder zu einer Zunahme des Auftretens, wobei typischerweise Patienten im 3. und 4. Lebensjahrzehnt in 80-90\% bilateral betroffen sind [36]. Die Therapie der Frühstadien umfasst eine disseminierte Koagulation der peripher ischämischen Areale. Es kommt sekundär zu einer Regression der sich an der Grenze zur Ischämie bildenden Neovaskularisationen ( $\bullet$ Abb. 15). Die Proliferationssegel, die in den weiter fortgeschrittenen Stadien mit einer ausgeprägten angiografischen Leckage assoziiert sein können, sind nicht direkt koagulierbar. Nach Anti-VEGF ist eine Regression möglich, aber der langfristige Erfolg muss durch die Koagulation der ischämischen Peripherie gesichert werden.

Eine ophthalmologisch vermutlich unterdiagnostizierte Erkrankung ist das Susac-Syndrom, eine durch den amerikanischen Neurologen John O. Susac 1975 erstbeschriebene „Retinocochleocerebral Vasculopathy“. Bis dato gibt es nur wenige Fallserien, jedoch ist die Prävalenz der Trias Enzephalopathie, retinale „Gefäßspasmen“ und Hörverlust (Tieftonschwerhörigkeit) ver- 

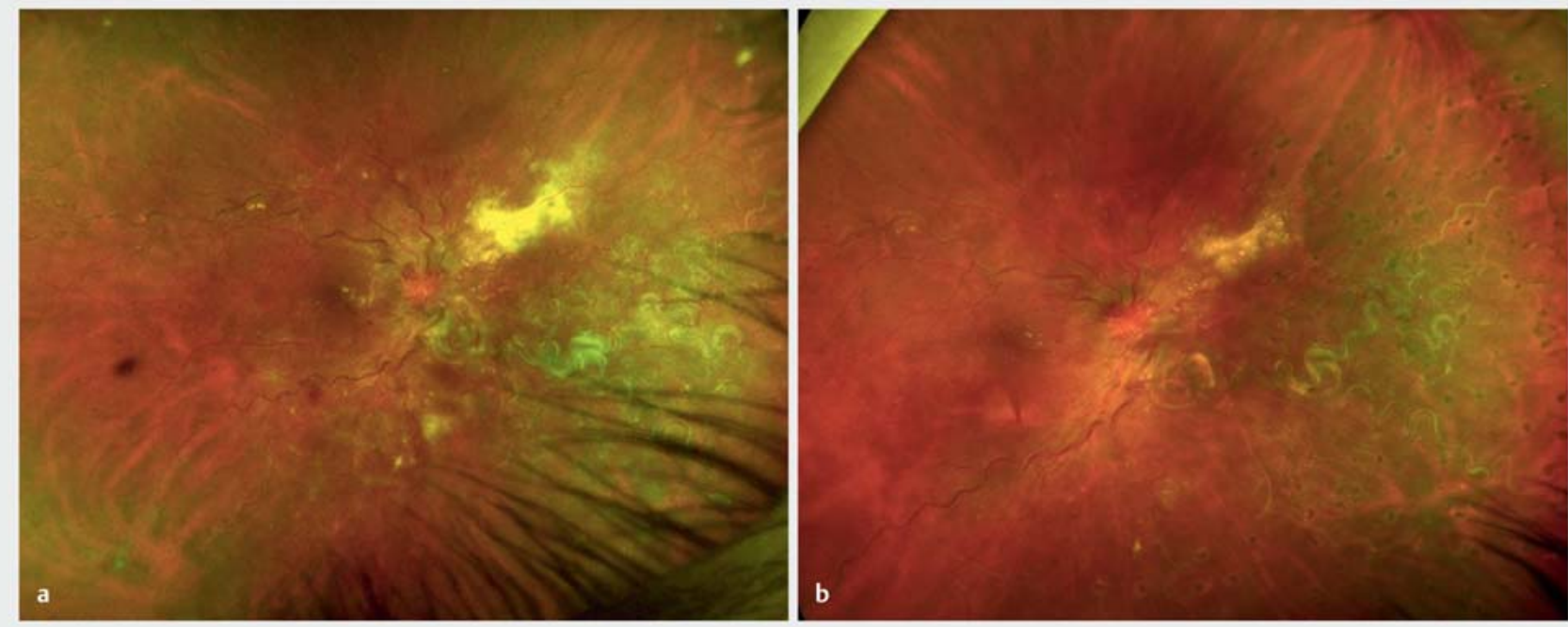

- Abb. 14 Racemöses Angiom bei Wyburn-Mason-Syndrom. Rechtes Auge mit den typischen korkenzieherartigen Gefäßveränderungen, hier insbesondere nach nasal inferior, und massiven Exsudationen nach nasal superior bei Z. n. zentralem Venenverschluss vor Therapie (a). b Nach intravitrealer Injektion von Bevacizumab und disseminierter Laserkoagulation ist ein deutlicher Rückgang der Exsudationen zu sehen.
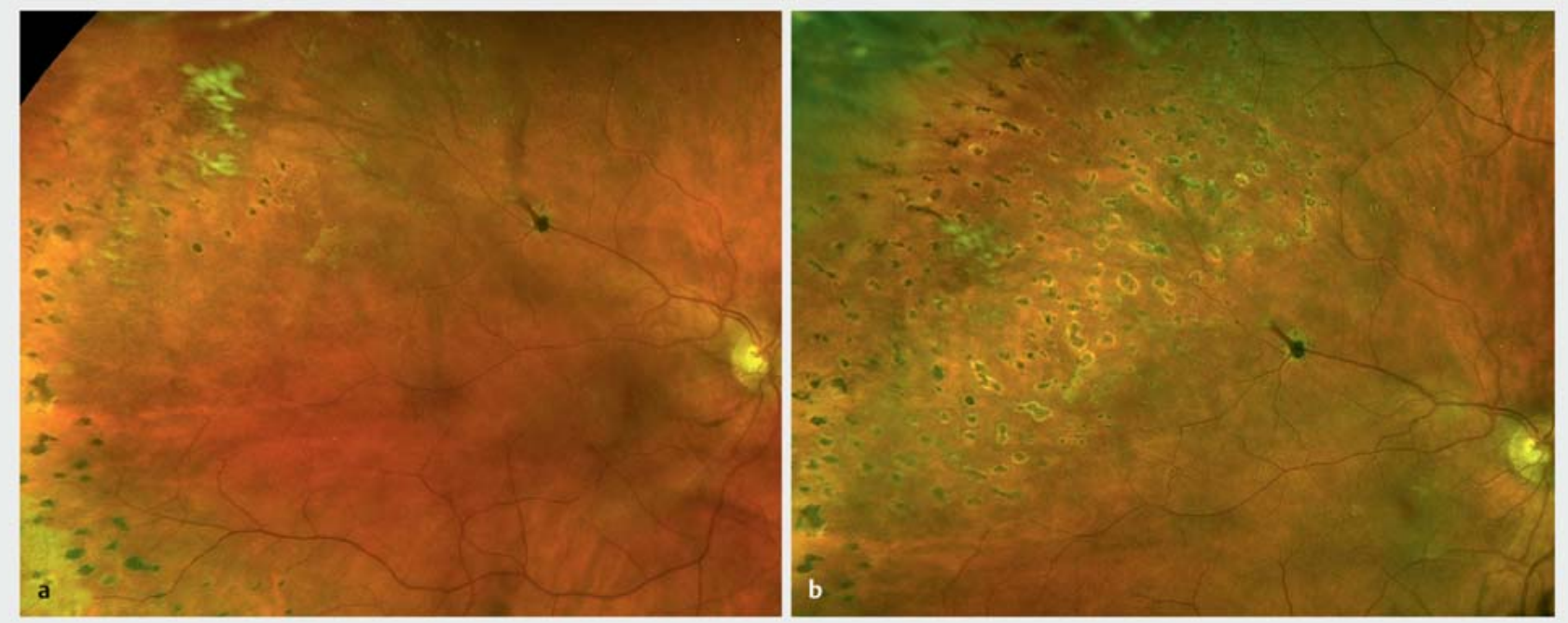

- Abb. 15 Morbus Eales. Linkes Auge mit Einscheidungen der peripheren Gefäße und Exsudation bei begonnener peripherer Laserkoagulation (a). Nach ergänzender disseminierter Laserkoagulation haben sich die Exsudationen deutlich zurückgebildet (b).

mutlich höher, und eine interdisziplinäre Kommunikation würde die Diagnosestellung erleichtern. Es wird eine autoimmune Genese der vasospastischen Episoden angenommen. Pathognomonisch sind angiografisch segmentale Leckagen und Gefäßverschlüsse in der Netzhautperipherie, in fortgeschrittenen Stadien auch bis in den peripapillären Bereich reichend ( $\bullet$ Abb. 16). Die Therapie richtet sich nach dem Ausmaß der systemischen Beteiligung und besteht in einer Immunsuppression, die gegebenenfalls mit einer Antikoagulation verbunden wird. Größere periphere Ischämien sollten disseminiert koaguliert werden in Analogie zu dem bei Morbus Eales beschriebenen Vorgehen. Allerdings gibt es keine Beschreibung von proliferativen Stadien bei Susac-Syndrom.

\section{Zusammenfassung}

Durch die multimodale Ultraweitwinkelbildgebung ergibt sich ein neuer Blick auf periphere vaskuläre Netzhauterkrankungen. Der Einsatz dieser Verfahren, und hier insbesondere der Angiografie, ist in der Differenzialdiagnose peripherer vaskulärer Netzhaut- 

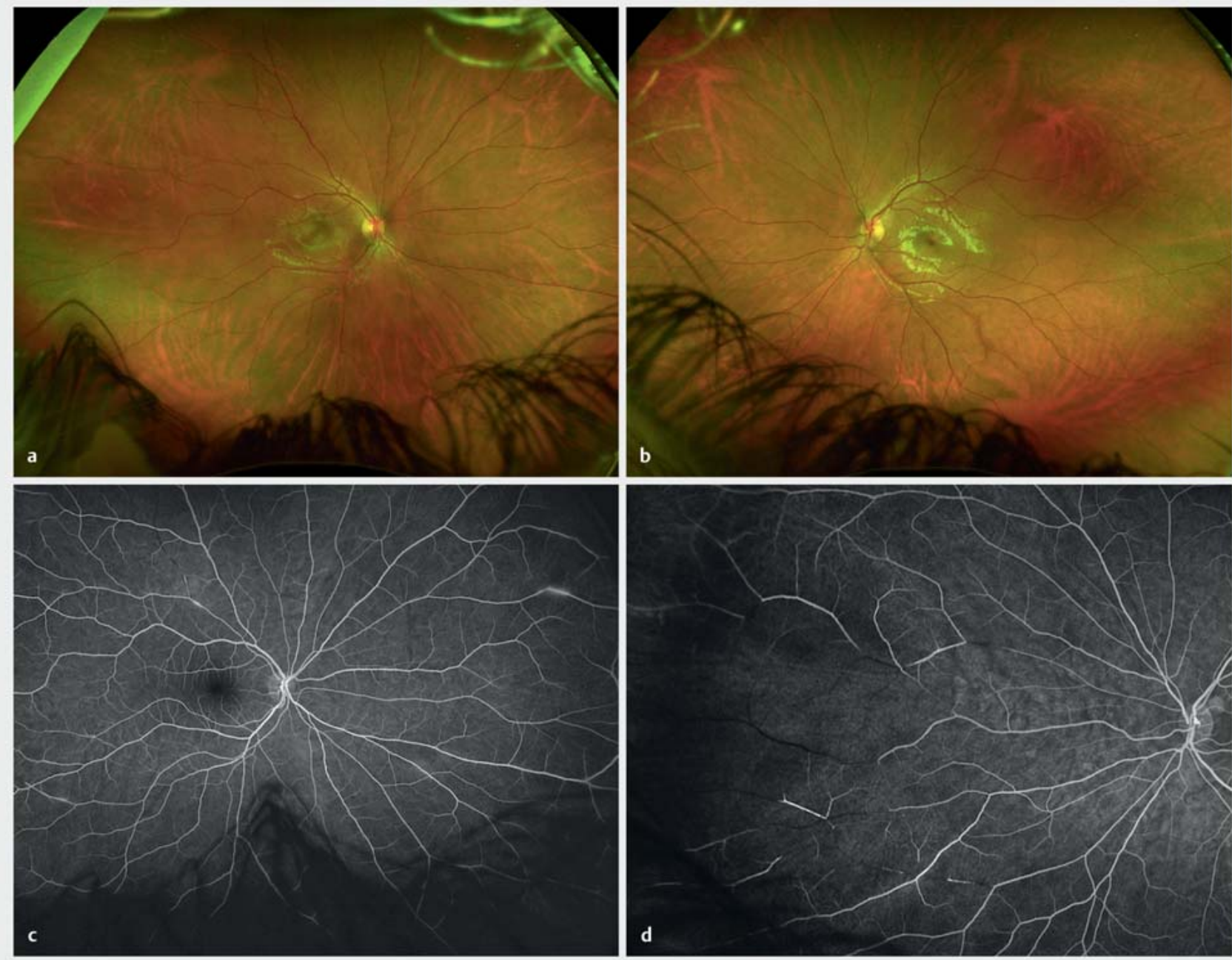

- Abb. 16 Susac-Syndrom. Beidseitiger Befund mit relativ unauffälligen Fundusfarbbildern (a und b), aber den charakteristischen segmentalen Leckagen und teils langstreckigen Gefäßverschlüssen in der Fluoreszenzangiografie (c und d).

erkrankungen nicht nur zur Dokumentation, sondern auch für die Therapieentscheidung und Verlaufsbeurteilung von Relevanz. Bei Erkrankungen wie Morbus Coats oder FEVR konnten mittels Ultraweitwinkelbildgebung Veränderungen in der Netzhautperipherie auf klinisch scheinbar nicht betroffenen Partneraugen festgestellt werden. Allerdings ist es auch mit aktuellen Ultraweitwinkelsystemen nicht möglich, verlässlich die gesamte Netzhaut abzubilden. Die Untersuchung muss immer gezielt erfolgen, um genau die Bereiche der Netzhautperipherie darzustellen, die betroffen sind. Daher bleibt auch in Zukunft die Funduskopie eine der wichtigsten Fertigkeiten der Augenärzte.

\section{Interessenkonflikt}

Die Autoren geben an, dass kein Interessenkonflikt besteht.

\section{Literatur}

[1] Lorenz B, Stieger K, Jäger M et al. Retinal Vascular Development With 0.312 mg Intravitreal Bevacizumab To Treat Severe Posterior Retinopathy Of Prematurity: A Longitudinal Fluorescein Angiographic Study. Retina 2017; 37: 97-111

[2] Kumar V, Chandra P, Kumar A. Ultra-wide field angiography in the management of Eales disease. Indian J Ophthalmol 2016; 64: 504-507

[3] Busch C, Löwen J, Pilger D et al. Quantification of radiation retinopathy after beam proton irradiation in centrally located choroidal melanoma. Graefes Arch Clin Exp Ophthalmol 2018. doi:10.1007/s00417-0184036-3

[4] Nürnberg D, Seibel I, Riechardt Al et al. Multimodale Bildgebung des Aderhautmelanoms mit seinen Differenzialdiagnosen, Therapie (Bestrahlungsplanung) und Verlaufskontrolle. Klin Monatsbl Augenheilkd 2018; 235: 1002-1013

[5] Mintz-Hittner HA, Kennedy KA, Chuang AZ. Efficacy of intravitreal bevacizumab for stage 3+ retinopathy of prematurity. N Engl J Med 2011; 364: 603-615 
[6] Mueller B, Salchow DJ, Waffenschmidt E et al. Treatment of type I ROP with intravitreal bevacizumab or laser photocoagulation according to retinal zone. Br J Ophthalmol 2017; 101: 365-370

[7] Hajrasouliha AR, Garcia-Gonzales JM, Shapiro MJ et al. Reactivation of Retinopathy of Prematurity Three Years After Treatment With Bevacizumab. Ophthalmic Surg Lasers Imaging Retina 2017; 48: 255-259

[8] Henaine-Berra A, Garcia-Aguirre G, Quiroz-Mercado H et al. Retinal fluorescein angiographic changes following intravitreal anti-VEGF therapy. J AAPOS 2014; 18: 120-123

[9] Lepore D, Quinn GE, Molle F et al. Follow-up to Age 4 Years of Treatment of Type 1 Retinopathy of Prematurity Intravitreal Bevacizumab Injection versus Laser: Fluorescein Angiographic Findings. Ophthalmology 2018; 125: $218-226$

[10] Geloneck MM, Chuang AZ, Clark WL et al. Refractive outcomes following bevacizumab monotherapy compared with conventional laser treatment: a randomized clinical trial. JAMA Ophthalmol 2014; 132: 13271333

[11] Hwang CK, Hubbard GB, Hutchinson AK et al. Outcomes after Intravitreal Bevacizumab versus Laser Photocoagulation for Retinopathy of Prematurity: A 5-Year Retrospective Analysis. Ophthalmology 2015; 122: 1008-1015

[12] Park KH, Hwang JM, Choi MY et al. Retinal detachment of regressed retinopathy of prematurity in children aged 2 to 15 years. Retina 2004; 24: $368-375$

[13] Joussen AM, Gordes RS, Heußen FA et al. [Retinal exudative disease in childhood: Coats' disease and familial exudative vitreoretinopathy (FEVR)]. Klin Monatsbl Augenheilkd 2013; 230: 902-913

[14] Kashani AH, Brown KT, Chang E et al. Diversity of retinal vascular anomalies in patients with familial exudative vitreoretinopathy. Ophthalmology 2014; 121: 2220-2227

[15] Spitznas M, Joussen F, Wessing A. Treatment of Coats' disease with photocoagulation. Albrecht Von Graefes Arch Klin Exp Ophthalmol 1976; 199: 31-37

[16] Shields CL, Shields JA, Barrett J et al. Vasoproliferative tumors of the ocular fundus. Classification and clinical manifestations in 103 patients. Arch Ophthalmol 1995; 113: 615-623

[17] Damato B. Vasoproliferative retinal tumour. Br J Ophthalmol 2006; 90: 399-400

[18] Heimann H, Bornfeld N, Vij O et al. Vasoproliferative tumours of the retina. Br J Ophthalmol 2000; 84: 1162-1169

[19] Irvine F, O’Donnell N, Kemp E et al. Retinal vasoproliferative tumors: surgical management and histological findings. Arch Ophthalmol 2000; 118: 563-569

[20] Smeets MH, Mooy CM, Baarsma GS et al. Histopathology of a vasoproliferative tumor of the ocular fundus. Retina 1998; 18: 470-472
[21] Hiscott P, Mudhar H. Is vasoproliferative tumour (reactive retinal glioangiosis) part of the spectrum of proliferative vitreoretinopathy? Eye (Lond) 2009; 23: 1851-1858

[22] Baines PS, Hiscott PS, McLeod D. Posterior non-vascularized proliferative extraretinopathy and peripheral nodular retinal telangiectasis. Trans Ophthalmol Soc U K 1982; 102: 487-491

[23] Laqua $\mathrm{H}$, Wessing A. Peripheral retinal telangiectasis in adults simulating a vascular tumor or melanoma. Ophthalmology 1983; 90: 1284-1291

[24] Bornfeld N. Vascular Tumors of the Retina. In: Joussen AM, ed. Retinal vascular Disease. Berlin, Heidelberg, New York: Springer; 2010: 446-449

[25] Anastassiou G, Bornfeld N, Schueler AO et al. Ruthenium-106 plaque brachytherapy for symptomatic vasoproliferative tumours of the retina. Br J Ophthalmol 2006; 90: 447-450

[26] Kitei PM, Say EA, Shields CL et al. Management of Retinal Vasoproliferative Tumor Associated with ROP by Plaque Brachytherapy. J Pediatr Ophthalmol Strabismus 2011; 48: e10-e12

[27] Brockmann C, Rehak M, Heufelder J et al. Predictors of treatment response of vasoproliferative retinal tumors to Ruthenium-106 brachytherapy. Retina 2016; 36: 2384-2390

[28] Seibel I, Nürnberg D, Löwen J et al. Peripher exsudative hämorrhagische Chorioretinopathie: Krankheitsverlauf, Diagnosestellung inklusive Weitwinkelbildgebung der assoziierten Erkrankungen altersabhängige Makuladegeneration und polypoidale Choriovaskulopathie, Therapie. Klin Monatsbl Augenheilkd 2018; 235: 995-1001

[29] Varshney N, Kebede AA, Owusu-Dapaah H et al. A Review of Von HippelLindau Syndrome. J Kidney Cancer VHL 2017; 4: 20-29

[30] Lonser RR, Glenn GM, Walther M et al. von Hippel-Lindau disease. Lancet 2003; 361: 2059-2067

[31] Nielsen SM, Rhodes L, Blanco I et al. Von Hippel-Lindau Disease: Genetics and Role of Genetic Counseling in a Multiple Neoplasia Syndrome. J Clin Oncol 2016; 34: 2172-2181

[32] Gaudric A, Krivosic V, Duguid G et al. Vitreoretinal surgery for severe retinal capillary hemangiomas in von Hippel-Lindau disease. Ophthalmology 2011; 118: 142-149

[33] Avci R, Yilmaz S, Inan UU et al. Vitreoretinal surgery for patients with severe exudative and proliferative manifestations of retinal capillary hemangioblastoma because of von Hippel-Lindau disease. Retina 2017; 37: 782-788

[34] Shah GK, Shields JA, Lanning RC. Branch retinal vein obstruction secondary to retinal arteriovenous communication. Am J Ophthalmol 1998; 126: $446-448$

[35] Elliot AJ. 30-year observation of patients with Eale's disease. Am J Ophthalmol 1975; 80: 404-408

[36] Das T, Pathengay A, Hussain N et al. Eales' disease: diagnosis and management. Eye (Lond) 2010; 24: 472-482 